Pacific Journal of Mathematics

CONTRACTION SEMIGROUPS IN LEBESGUE SPACE 


\title{
CONTRACTION SEMIGROUPS IN LEBESGUE SPACE
}

\author{
RYOTARO SATO
}

\begin{abstract}
Let $\left(T_{t}: t>0\right)$ be a strongly continuous semigroup of linear contractions on $L_{1}(X, \boldsymbol{\Sigma}, \mu)$, where $(X, \boldsymbol{\Sigma}, \mu)$ is a $\sigma$ finite measure space. Without assuming the initial continuity of the semigroup it is shown that $\left(T_{t}: t>0\right)$ is dominated by a strongly continuous semigroup $\left(S_{t}: t>0\right)$ of positive linear contractions on $L_{1}(X, \Sigma, \mu)$, i.e., that $\left|T_{t} f\right| \leqq S_{t}|f|$ holds a.e. on $X$ for all $f \in L_{1}(X, \Sigma, \mu)$ and all $t>0$. As an application, a representation of $\left(T_{t}: t>0\right)$ in terms of $\left(S_{t}: t>0\right)$ is obtain$e d$, and the question of the almost everywhere convergence of $1 / b \int_{0}^{b} T_{t} f d t$ as $b \rightarrow+0$ is considered.
\end{abstract}

Introduction. Let $(X, \Sigma, \mu)$ be a $\sigma$-finite measure space and let $L_{p}(X)=L_{p}(X, \Sigma, \mu), 1 \leqq p \leqq \infty$, be the usual Banach spaces of real or complex functions on $(X, \Sigma, \mu)$. For a set $A \in \Sigma, L_{p}(A)$ denotes the Banach space of all $L_{p}(X)$-functions that vanish a.e. on $X-A$. If $f \in L_{p}(X)$, we define supp $f$ to be the set of all $x \in X$ at which $f(x) \neq 0$. Relations introduced below are assumed to hold modulo sets of measure zero. A linear operator $T$ on $L_{p}(X)$ is called a contraction if $\|T\|_{p} \leqq 1$, and positive if $f \geqq 0$ implies $T f \geqq 0$.

Let $\left(T_{t}: t>0\right)$ be a strongly continuous semigroup of linear contractions on $L_{1}(X)$, i.e.,

(i) each $T_{t}$ is a linear contraction on $L_{1}(X)$,

(ii) $T_{t} T_{s}=T_{t+s}$ for all $t, s>0$,

(iii) for every $f \in L_{1}(X)$ and every $s>0, \lim _{t \rightarrow s}\left\|T_{t} f-T_{s} f\right\|_{1}=0$.

Under the additional hypothesis of strong-lim $\lim _{t \rightarrow 0} T_{t}=I$ ( $I$ denotes the identity operator), Kubokawa [6] proved that there exists a strongly continuous semigroup $\left(S_{t}: t>0\right)$ of positive linear contractions on $L_{1}(X)$ such that $\left|T_{t} f\right| \leqq S_{t}|f|$ a.e. on $X$ for all $f \epsilon$ $L_{1}(X)$ and all $t>0$. The main purpose of this paper is to prove the same result, without assuming any additional hypothesis. We then obtain a representation of $\left(T_{t}: t>0\right)$ in terms of $\left(S_{t}: t>0\right)$ which is a continuous extension of Akcoglu-Brunel's representation ([1], Theorem 3.1), and a decomposition of the space $X$ for $\left(T_{t}: t>0\right)$ which asserts the existence of a set $Y \in \Sigma$ such that $T_{t} f \in L_{1}(Y)$ for all $f \in L_{1}(X)$ and all $t>0$ and also such that if $f \in L_{1}(Y)$ then $T_{t} f$ converges in the norm topology of $L_{1}(X)$ as $t \rightarrow+0$ and $1 / b \int_{0}^{b} T_{t} f d t$ converges a.e. on $X$ as $b \rightarrow+0$. 
Existence theorem. Our main result is the following existence theorem.

THEOREM 1. If $\left(T_{t}: t>0\right)$ is a strongly continuous semigroup of linear contractions on $L_{1}(X)$, then there exists a strongly continuous semigroup $\left(S_{t}: t>0\right)$ of positive linear contractions on $L_{1}(X)$, called the semigroup modulus of $\left(T_{t}: t>0\right)$, such that

$$
\left|T_{t} f\right| \leqq S_{t}|f| \quad\left(f \in L_{1}(X), t>0\right) .
$$

If $0 \leqq f \in L_{1}(X), S_{t}$ is given by

$$
S_{t} f=\sup \left\{\tau_{t_{1}} \cdots \tau_{t_{n}} f: \sum_{i=1}^{n} t_{i}=t, t_{i}>0, n \geqq 1\right\}
$$

where $\tau_{t}$ denotes the linear modulus of $T_{t}$ in the sense of ChaconKrengel ([3]).

Proof. For $0 \leqq f \in L_{1}(X)$ and $t>0$, put

$$
M(t, f)=\left\{\tau_{t_{1}} \cdots \tau_{t_{n}} f: \sum_{i=1}^{n} t_{i}=t, t_{i}>0, n \geqq 1\right\} .
$$

Since $\left\|\tau_{t}\right\|_{1}=\left\|T_{t}\right\|_{1} \leqq 1$ and $\tau_{t} \tau_{s} f \geqq \tau_{t+s} f$ for all $t, s>0$, we see that if $g_{1}$ and $g_{2}$ are in $M(t, f)$, then there exists a function $h$ in $M(t, f)$ such that

$$
\max \left(g_{1}, g_{2}\right) \leqq h \text { and }\|h\|_{1} \leqq\|f\|_{1} \text {. }
$$

Thus it is possible to define a function $S_{t} f$ in $L_{1}(X)$ by the relation:

$$
S_{t} f=\sup \{g: g \in M(t, f)\} \text {. }
$$

It is clear that $\left\|S_{t} f\right\|_{1} \leqq\|f\|_{1}$ and $S_{t} f \geqq 0$. It is easily seen that if $c$ is a positive constant and $f$ and $g$ are nonnegative functions in $L_{1}(X)$, then

$$
S_{t}(c f)=c S_{t} f \text { and } S_{t}(f+g)=S_{t} f+S_{t} g .
$$

Therefore $S_{t}$ may be regarded as a positive linear contraction on $L_{1}(X)$. By the definition of $S_{t}$ it follows that

$$
S_{t} S_{s}=S_{t+s} \quad(t, s>0) .
$$

It is now enough to prove the strong continuity of $\left(S_{t}: t>0\right)$. To do this, we first show the following result:

$$
\lim _{t \rightarrow s+0}\left\|\tau_{t} f-\tau_{s} f\right\|_{1}=0 \quad\left(f \in L_{1}(X), s>0\right) .
$$

To see this, we may and do assume without loss of generality 
that $f$ is nonnegative. Let $\varepsilon>0$ be given. By [3] there exist functions $g_{i} \in L_{1}(X), 1 \leqq i \leqq n$, such that

$$
\left|g_{i}\right| \leqq f \text { and }\left\|\tau_{s} f-\max _{1 \leqq i \leqq n}\left|T_{s} g_{i}\right|\right\|_{1}<\varepsilon \text {. }
$$

Since $\left(T_{t}: t>0\right)$ is strongly continuous on $(0, \infty)$, we can take a $\delta>0$ so that $|s-t|<\delta$ implies $\left\|T_{s} g_{i}-T_{t} g_{i}\right\|_{1}<\varepsilon / n$ for each $1 \leqq$ $i \leqq n$. Fix a $t>0$ so that $|s-t|<\delta$. We then have $\|\left|T_{s} g_{i}\right|-$ $\left|T_{t} g_{i}\right|\left\|_{1} \leqq\right\| T_{s} g_{i}-T_{t} g_{i} \|_{1}<\varepsilon / n$ for each $1 \leqq i \leqq n$, and so it follows that

$$
\left\|\tau_{s} f-\max _{1 \leqq i \leqq n}\left|T_{t} g_{i}\right|\right\|_{1}<2 \varepsilon
$$

By this and the fact that $\tau_{t} f \geqq \max _{1 \leqq i \leqq n}\left|T_{t} g_{i}\right|$, we get

$$
\left\|\left(\tau_{t} f-\tau_{s} f\right)^{-}\right\|_{1} \leqq\left\|\left(\max _{1 \leqq i \leqq n}\left|T_{t} g_{i}\right|-\tau_{s} f\right)^{-}\right\|_{1}<2 \varepsilon
$$

Therefore

$$
\lim _{t \rightarrow s}\left\|\left(\tau_{t} f-\tau_{s} f\right)^{-}\right\|_{1}=0 .
$$

Next, let $t>s$ and write $t=s+a$. Since

$$
\left\|\left(\tau_{a} \tau_{s} f-\tau_{s} f\right)^{-}\right\|_{1} \leqq\left\|\left(\tau_{t} f-\tau_{s} f\right)^{-}\right\|_{1},
$$

it follows that

$$
\lim _{a \rightarrow+0}\left\|\left(\tau_{a} \tau_{s} f-\tau_{s} f\right)^{-}\right\|_{1}=0
$$

On the other hand,

$$
\tau_{a} \tau_{s} f=\left(\tau_{a} \tau_{s} f-\tau_{s} f\right)^{+}-\left(\tau_{a} \tau_{s} f-\tau_{s} f\right)^{-}+\tau_{s} f .
$$

Thus, by (5), we have that

$$
\begin{aligned}
\left\|\left(\tau_{a+s} f-\tau_{s} f\right)^{+}\right\|_{1} & \leqq\left\|\left(\tau_{a} \tau_{s} f-\tau_{s} f\right)^{+}\right\|_{1} \\
& \leqq\left\|\left(\tau_{a} \tau_{s} f-\tau_{s} f\right)^{-}\right\|_{1}+\left\|\tau_{a} \tau_{s} f\right\|_{1}-\left\|\tau_{s} f\right\|_{1} \\
& \leqq\left\|\left(\tau_{a} \tau_{s} f-\tau_{s} f\right)^{-}\right\|_{1} \rightarrow 0
\end{aligned}
$$

as $a \rightarrow+0$, because $\left\|\tau_{a}\right\|_{1} \leqq 1$. This and (4) establish (3).

We next show that

$$
\lim _{t \rightarrow s+0}\left\|S_{t} f-S_{s} f\right\|_{1}=0 \quad\left(f \in L_{1}(X), s>0\right) .
$$

To see this, we may and do assume without loss of generality that $f$ is nonnegative. Let $\varepsilon>0$ be given, and choose a function $g \in M(s, f)$ so that 


$$
\left\|S_{s} f-g\right\|_{1}<\varepsilon,
$$

where $g$ is of the form

$$
g=\tau_{t_{1}} \cdots \tau_{t_{n}} f, \sum_{i=1}^{n} t_{i}=s, \text { and } t_{i}>0(1 \leqq i \leqq n) .
$$

Let $s_{n}>t_{n}$. Then

$$
\begin{aligned}
\left\|g-\tau_{t_{1}} \cdots \tau_{t_{n-1}} \tau_{s_{n}} f\right\|_{1} & =\left\|\tau_{t_{1}} \cdots \tau_{t_{n-1}}\left(\tau_{t_{n}} f-\tau_{s_{n}} f\right)\right\|_{1} \\
& \leqq\left\|\tau_{t_{n}} f-\tau_{s_{n}} f\right\|_{1},
\end{aligned}
$$

and hence, by (3),

$$
\lim _{s_{n} \rightarrow t_{n}+0}\left\|g-\tau_{t_{1}} \cdots \tau_{t_{n-1}} \tau_{s_{i n}} f\right\|_{1}=0 .
$$

Let us write $t=t_{1}+\cdots+t_{n-1}+s_{n}(>s)$. Since

$$
S_{t} f-S_{s} f \geqq\left(\tau_{t_{1}} \cdots \tau_{t_{n-1}} \tau_{s_{n}} f-g\right)+\left(g-S_{s} f\right),
$$

it follows that

$$
\left(S_{t} f-S_{s} f\right)^{-} \leqq\left|\tau_{t_{1}} \cdots \tau_{t_{n-1}} \tau_{s_{n}} f-g\right|+\left|g-S_{s} f\right| .
$$

This and (7) yield that

$$
\limsup _{t \rightarrow s ;-0}\left\|\left(S_{t} f-S_{s} f\right)^{-}\right\|_{1} \leqq \varepsilon .
$$

Since $\varepsilon$ is arbitrary,

$$
\lim _{t \rightarrow s \neq n}\left\|\left(S_{t} f-S_{\varepsilon} f\right)^{-}\right\|_{1}=0 .
$$

Hence

$$
\begin{aligned}
\left\|\left(S_{t} f-S_{s} f\right)^{+}\right\|_{1} & =\left\|\left(S_{t} f-S_{s} f\right)^{-}\right\|_{1}+\left\|S_{t} f\right\|_{1}-\left\|S_{s} f\right\|_{1} \\
& \leqq\left\|\left(S_{t} f-S_{s} f\right)^{-}\right\|_{1} \longrightarrow 0
\end{aligned}
$$

as $t \rightarrow s+0$, because $\left\|S_{t} f\right\|_{1} \leqq\left\|S_{s} f\right\|_{1}$ for all $t>s$. This proves (6).

Using (6), it is now direct to show that the semigroup $\left(S_{t}: t>0\right)$ is strongly continuous on $(0, \infty)$, and we omit the details.

TheOREm 2. Let $\left(T_{t}: t>0\right)$ and $\left(S_{t}: t>0\right)$ be as in Theorem 1. Then $T_{t}$ converges strongly as $t \rightarrow+0$ if and only if $S_{t}$ converges strongly as $t \rightarrow+0$.

Proof. If $T_{0}=$ strong- $\lim _{t \rightarrow+0} T_{t}$ exists, then $\left(T_{t}: t \geqq 0\right)$ is a semigroup and strongly continuous on $[0, \infty)$. Hence we can apply the same arguments as in the proof of Theorem 1 to obtain that $\lim _{t \rightarrow+0}\left\|S_{t} f-\tau_{0} f\right\|_{1}=0$ for all $f \in L_{1}(X)$, where $\tau_{0}$ denotes the linear modulus of $T_{0}$. 
Conversely, if $S_{0}=$ strong-lim $\lim _{t \rightarrow 0} S_{t}$ exists, then, for all $f \in L_{1}(X)$, the set $\left\{T_{t} f: 0<t<1\right\}$ is weakly sequentially compact in $L_{1}(X)$, since $\left|T_{t} f\right| \leqq S_{t}|f|$ and $\lim _{t \rightarrow+0}|| S_{t}|f|-S_{0}|f| \|_{1}=0$ (cf. Theorem IV. 8.9 in [4]). Thus, by Lemma 1 of the author [8], $T_{t}$ converges strongly as $t \rightarrow+0$.

The hypothesis of being a contraction semigroup can not be weakened in Theorem 1 . To see this, we give the following example, motivated by $\mathrm{S}$. Tsurumi.

ExAMPLE. Let $X$ be the positive integers, $\Sigma$ all possible subsets of $X$, and $\mu$ the counting measure. Let $\varepsilon>0$ be given. By an elementary computation, there exists a real constant $r$, with $1 / e<$ $r<1$, such that

$$
1<\sup \left\{r^{t}(|\cos t|+|\sin t|): t \geqq 0\right\}<1+\varepsilon .
$$

For $f \in L_{1}(X)$ and $t>0$, define

$$
T_{t} f(2 n-1)=r^{n t}[f(2 n-1) \cos n t-f(2 n) \sin n t] \quad(n \geqq 1)
$$

and

$$
T_{t} f(2 n)=r^{n t}[f(2 n-1) \sin n t+f(2 n) \cos n t] \quad(n \geqq 1) .
$$

It is easily seen that $\left(T_{t}: t>0\right)$ is a strongly continuous semigroup of linear operators on $L_{1}(X)$ satisfying $\left\|T_{t}\right\|_{1} \leqq 1+\varepsilon$ for all $t>0$. Furthermore

$$
\lim _{m \rightarrow \infty}\left\|\left(\tau_{1 / m}\right)^{m}\right\|_{1}=\infty \text {. }
$$

To see this, let $1_{n}$ denote the indicator function of $\{n\}$. Then

$$
\begin{aligned}
\left\|\left(\tau_{1 / m}\right)^{m}\right\|_{1} & \geqq\left\|\left(\tau_{1 / m}\right)^{m}\left(1_{2 n-1}+1_{2 n}\right)\right\|_{1} /\left\|1_{2 n-1}+1_{2 n}\right\|_{1} \\
& =\left[r^{n / m}\left(\left|\cos \frac{n}{m}\right|+\left|\sin \frac{n}{m}\right|\right)\right]^{m} \quad(n \geqq 1),
\end{aligned}
$$

as has been pointed out by S. Koshi. Hence (8) implies (9).

By (9) it is now immediate to see that $\left(T_{t}: t>0\right)$ can not be dominated by a semigroup of positive linear operators on $L_{1}(X)$.

Representation theorem. Let $\left(T_{t}: t>0\right)$ be a strongly continuous semigroup of linear contractions on $L_{1}(X)$. It is well known that given an $f \in L_{1}(X)$ there exists a scalar function $g(t, x)$ on $(0$, $\infty) \times X$, measurable with respect to the product of Lebesgue measure and $\mu$, such that for each $t>0, g(t, x)$, as a function of $x$, belongs to the equivalence class of $T_{t} f$. In the sequel $g(t, x)$ will 
be denoted by $T_{t} f(x)$. Using Fubini's theorem, we see that there exists a set $E(f) \in \Sigma$, with $\mu(E(f))=0$, such that if $x \notin E(f)$ then the scalar function $t \mapsto T_{t} f(x)$ is Lebesgue integrable on every finite interval $(a, b)$ and the integral $\int_{t}^{b} T_{t} f(x) d t$, as a function of $x$, belongs to the equivalence class of $\int_{a}^{b} T_{t} f d t$, where $\int_{a}^{b} T_{t} f d t$ denotes the Bochner integral of the vector valued function $t \mapsto T_{t} f$ with respect to Lebesgue measure on $(a, b)$.

If $\left(S_{t}: t>0\right)$ denotes the semigroup modulus of $\left(T_{t}: t>0\right)$, then the ratio ergodic theorem holds for $\left(S_{t}: t>0\right)$, i.e., for any $f$ and $g$ in $L_{1}(X)$, with $g \geqq 0$, the ratio ergodic limit

$$
\lim _{b \rightarrow \infty}\left(\int_{0}^{b} S_{t} f(x) d t\right) /\left(\int_{0}^{b} S_{t} g(x) d t\right)
$$

exists and is finite a.e. on the set $\left\{x: \int_{0}^{\infty} S_{t} g(x) d t>0\right\}$ (cf. [5]). Thus Hopf's decomposition holds, i.e., $X$ decomposes into two measurable sets $C$ and $D$, called respectively the conservative and dissipative parts of $X$, such that if $0 \leqq g \in L_{1}(X)$ then $\int_{0}^{\infty} S_{t} g(x) d t=\infty$ or 0 a.e. on $C$ and $\int_{0}^{\infty} S_{t} g(x) d t<\infty$ a.e. on $D$. A set $A \in \Sigma$ is called invariant (under $\left(S_{t}: t>0\right)$ ), if $S_{t} L_{1}(A) \subset L_{1}(A)$ for all $t>0$. It is immediate that $A$ is invariant under $\left(S_{t}: t>0\right)$ if and only if it is invariant under $\left(T_{t}: t>0\right)$. It is known (cf. [7]) that $C$ is invariant and the class $\Sigma_{i}$ of all invariant subsets of $C$ forms a $\sigma$-field in the class of all measurable subsets of $C$.

We are now in a position to state our representation theorem.

THEOREM 3. Let $\left(T_{t}: t>0\right)$ be a strongly continuous semigroup of linear contractions on $L_{1}(X)$ and $\left(S_{t}: t>0\right)$ denote the semigroup modulus of $\left(T_{t}: t>0\right)$. Let $C$ denote the conservative part of $X$ with respect to $\left(S_{t}: t>0\right)$ and let $\Sigma_{i}$ be the $\sigma$-field of invariant subsets of $C$. Then there exists a (unique) set $\Gamma \in \Sigma_{i}$ and a function $u \in L_{\infty}(\Gamma)$ such that

(i) $|u|=1$ a.e. on $\Gamma$ and $T_{t} f=(1 / u) S_{t}(u f)$ for all $f \in L_{1}(\Gamma)$ and all $t>0$,

(ii) if $\Delta=C-\Gamma$, then the closed linear hull of $\left\{f-T_{t} f\right.$ : $\left.f \in L_{1}(\Delta), t>0\right\}$ is $L_{1}(\Delta)$,

(iii) a function $v \in L_{\infty}(\Gamma)$, with $|v|>0$ a.e. on $\Gamma$, satisfies $T_{t} f=(1 / v) S_{t}(v f)$ for all $f \in L_{1}(\Gamma)$ and all $t>0$ if and only if there exists a function $r \in L_{\infty}(\Gamma)$ such that $|r|>0$ a.e. on $\Gamma, S_{t}^{*} r=$ $r$ a.e. on $\Gamma$ for all $t>0$, and $v=r u$.

Proof. Let $h \in L_{\infty}(C)$ be such that $T_{t}^{*} h=h$ a.e. on $C$ for all $t>0$. Since $|h|=\left|T_{t}^{*} h\right| \leqq \tau_{t}^{*}|h| \leqq S_{t}^{*}|h|$ and the conservative part 
of $X$ with respect to each single operator $S_{t}$ is exactly $C$ (cf. [7]), it follows that $|h|=S_{t}^{*}|h|$ a.e. on $C$ for all $t>0$, and hence supp $h \in \Sigma_{i}$. By this, we can find a function $h \in L_{\infty}(C)$ such that $T_{t}^{*} h=h$ a.e. on $C$ for all $t>0$ and also such that if $f \in L_{\infty}(C)$ satisfies $T_{t}^{*} f=f$ a.e. on $C$ for all $t>0$, then supp $f \subset \operatorname{supp} h$. Put $\Gamma=$ supp $h$ and define $u \in L_{\infty}(\Gamma)$ by $u=h /|h|$ a.e. on $\Gamma$. If $0 \leqq f \in L_{1}(\Gamma)$ and $t>0$, then, as in [1],

$$
\begin{gathered}
\int\left(S_{t} f\right)|h| d \mu=\int f S_{t}^{*}|h| d \mu=\int f|h| d \mu=\int(f / u) h d \mu \\
=\int(f / u) T_{t}^{*} h d \mu=\int T_{t}(f / u) u|h| d \mu .
\end{gathered}
$$

Hence $S_{t} f=T_{t}(f / u) u$, since $S_{t} f \geqq\left|T_{t}(f / u)\right|=\left|T_{t}(f / u) u\right|$, and (i) is established.

To prove (ii), let $h \in L_{\infty}(\Delta)$ be such that $\int\left(f-T_{t} f\right) h d \mu=0$ for all $f \in L_{1}(\Delta)$ and all $t>0$. Then $T_{t}^{*} h=h$ a.e. on $\Delta$ (and hence on $C)$ for all $t>0$. Therefore, by the definition of $\Gamma, h=0$ a.e. on $\Delta$, and (ii) follows from the Hahn-Banach theorem.

To prove (iii), let $v \in L_{\infty}(\Gamma)$ and $|v|>0$ a.e. on $\Gamma$. Put $r=v / u$. Then $T_{t} f=(1 / v) S_{t}(v f)$ for all $f \in L_{1}(\Gamma)$ and all $t>0$ if and only if $(1 / r \cdot u) S_{t}(r u f)=(1 / u) S_{t}(u f)$ for all $f \in L_{1}(\Gamma)$ and all $t>0$, or equivalently, $S_{t}(r f)=r S_{t} f$ for all $f \in L_{1}(\Gamma)$ and all $t>0$, since $\{u f$ : $\left.f \in L_{1}(\Gamma)\right\}=L_{1}(\Gamma)$. And this is equivalent to the fact that $S_{t}^{*} r=r$ a.e. on $\Gamma$ for all $t>0$, by Lemma 2.4 in [1].

The proof is complete.

Decomposition theorem. It is shown that, after eliminating an uninteresting subset of $X$, a strongly continuous semigroup $\left(T_{t}\right.$ : $t>0$ ) of linear contractions on $L_{1}(X)$ can be made strongly continuous at the origin and the local ergodic theorem holds.

THEOREM 4. Let $\left(T_{t}: t>0\right)$ be a strongly continuous semigroup of linear contractions on $L_{1}(X)$. Then $X$ can be written as the union of two disjoint measurable sets $Y$ and $Z$ with the following properties:

(i) For every $f \in L_{1}(X)$ and every $t>0, T_{t} f \in L_{1}(Y)$.

(ii) For every $f \in L_{1}(Y), T_{t} f$ converges in the norm topology of $L_{1}(X)$ as $t \rightarrow+0$ and

$$
\lim _{b \rightarrow+0} \frac{1}{b} \int_{0}^{b} T_{t} f(x) d t
$$

exists a.e. on $X$.

(iii) For every $f \in L_{1}(Y)$ with $f>0$ a.e. on $Y$, 


$$
Y=\bigcup_{n=1}^{\infty}\left\{x: \tau_{1 / n} f(x)>0\right\} .
$$

Proof. Let $\left(S_{t}: t>0\right)$ be the semigroup modulus of $\left(T_{t}: t>0\right)$. Fix an $h \in L_{1}(X)$ with $h>0$ a.e. on $X$, and put

$$
Y=\bigcup_{n=1}^{\infty}\left\{x: S_{1 / n} h(x)>0\right\}
$$

and $Z=X-Y$. It is easily seen that $S_{t} f \in L_{1}(Y)$ and hence $T_{t} f \in$ $L_{1}(Y)$ for all $f \in L_{1}(X)$ and all $t>0$. If we write $h_{0}=\int_{0}^{1} S_{t} h d t$, then $h_{0} \in L_{1}(Y), h_{0}>0$ a.e. on $Y$, and $\lim _{t \rightarrow+0}\left\|S_{t} h_{0}-h_{0}\right\|_{1}=0$. Therefore, by approximation, the set $\left\{S_{t} f: 0<t<1\right\}$ is weakly sequentially compact in $L_{1}(X)$ for all $0 \leqq f \in L_{1}(Y)$, from which we observe that the set $\left\{T_{t} f: 0<t<1\right\}$ is also weakly sequentially compact in $L_{1}(X)$ for all $f \in L_{1}(Y)$, since $\left|T_{t} f\right| \leqq S_{t}|f|$ for all $t>0$. Hence Lemma 1 of the author [8] implies that $T_{t} f$ converges in the norm topology of $L_{1}(X)$ as $t \rightarrow+0$ for all $f \in L_{1}(Y)$.

To prove the second part of (ii), we may and do assume without loss of generality that $X=Y$. Put $T_{0}=$ strong- $\lim _{t \rightarrow+0} T_{t}$, and let $f \in L_{1}(X)$. Then $f$ can be written as $f=g+h$, where $g=T_{0} f$ and $T_{t} h=0$ for all $t \geqq 0$, because $T_{t} T_{0}=T_{0} T_{t}=T_{t}$ for all $t \geqq 0$. It follows that

$$
\lim _{a \rightarrow+0}\left\|(f-h)-\frac{1}{a} \int_{0}^{a} T_{t} g d t\right\|_{1}=0 .
$$

If we write $f_{a}=h+1 / a \int_{0}^{a} T_{t} g d t$, then it is easily seen that

$$
\lim _{b \rightarrow+0} \frac{1}{b} \int_{0}^{b} T_{t} f_{a}(x) d t=f_{a}(x)-h(x) \text { a.e. }
$$

on $X$. On the other hand, by Akcoglu-Chacon's local ergodic theorem ([2]),

$$
\sup _{0<b<1}\left|\frac{1}{b} \int_{0}^{b} T_{t} f(x) d t\right| \leqq \sup _{0<b<1} \frac{1}{b} \int_{0}^{b} S_{t}|f|(x) d t<\infty \quad \text { a.e. }
$$

on $X$. Thus, the second part of (ii) follows from Banach's convergence theorem (cf. Theorem IV. 11. 3 in [4]).

For the proof of (iii), let $f \in L_{1}(Y), f>0$ a.e. on $Y$. Put

$$
P=\bigcup_{n=1}^{\infty}\left\{x: \tau_{1 / n} f(x)>0\right\} \text {. }
$$

Clearly, $P \subset Y$, and by the definition of $Y$ and (i),

$$
Y=\bigcup_{n=1}^{\infty}\left\{x: S_{1 / n} f(x)>0\right\} \text {. }
$$


Let $1 / n<t$. Then $\tau_{t} f \leqq \tau_{1 / n} \tau_{t-(1 / n)} f$, and so supp $\tau_{t} f \subset \operatorname{supp} \tau_{1 / n} f$. Thus it follows that

$$
\operatorname{supp} S_{t} f \subset P \quad(t>0) .
$$

Therefore $Y \subset P$, and (iii) is established.

The proof is complete.

In conclusion, the author would like to remark that the question of whether the almost everywhere convergence of $1 / b \int_{0}^{b} T_{t} f(x) d t$ as $b \rightarrow+0$ holds for all $f \in L_{1}(Z)$ remains an open problem.

\section{REFERENCES}

1. M. A. Akcoglu and A. Brunel, Contractions on $L_{1}$-spaces, Trans. Amer. Math. Soc., 155 (1971), 315-325.

2. M. A. Akcoglu and R. V. Chacon, A local ratio theorem, Canad. J. Math., 22 (1970), 545-552.

3. R. V. Chacon and U. Krengel, Linear modulus of a linear operator, Proc. Amer. Math. Soc., 15 (1964), 553-559.

4. N. Dunford and J. T. Schwartz, Linear Operators, Part I: General Theory, Interscience Publishers, Inc., New York, 1958.

5. H. Fong and L. Sucheston, On the ratio ergodic theorem for semigroups, Pacific

J. Math., 39 (1971), 659-667.

6. Y. Kubokawa, Ergodic theorems for contraction semigroups, J. Math. Soc. Japan, 27 (1975), 184-193.

7. M. Lin, Semigroups of Markov operators, Boll. Un. Mat. Ital., (4), 6 (1972), 20-44.

8. R. Sato, A note on a local ergodic theorem, Comment. Math. Univ. Carolinae, 16 (1975), 1-11.

Received July 27, 1977.

JOSAI UNIVERSITY

SAKado, SaItAMa, 350-02 JAPAN 



\section{PACIFIC JOURNAL OF MATHEMATICS}

\section{EDITORS}

RICHARD ARENS (Managing Editor)

University of California

Los Angeles, California 90024

C. W. CurTis

University of Oregon

Eugene, OR 97403

C. C. MOORE

University of California

Berkeley, CA 94720
J. DUGUNDJI

Department of Mathematics University of Southern California Los Angeles, California 90007

R. FinN AND J. Milgram Stanford University Stanford, California 94305

\section{ASSOCIATE EDITORS}

E. F. BECKENBACH

B. H. NeumanN

F. WOLF

K. YoSHIDA

\section{SUPPORTING INSTITUTIONS}

UNIVERSITY OF BRITISH COLUMBIA CALIFORNIA INSTITUTE OF TECHNOLOGY UNIVERSITY OF CALIFORNIA MONTANA STATE UNIVERSITY UNIVERSITY OF NEVADA, RENO NEW MEXICO STATE UNIVERSITY OREGON STATE UNIVERSITY UNIVERSITY OF OREGON
UNIVERSITY OF SOUTHERN CALIFORNIA

STANFORD UNIVERSITY

UNIVERSITY OF HAWAII

UNIVERSITY OF TOKYO

UNIVERSITY OF UTAH

WASHINGTON STATE UNIVERSITY

UNIVERSITY OF WASHINGTON 


\section{Pacific Journal of Mathematics \\ Vol. 78, No. $1 \quad$ March, 1978}

Simeon M. Berman, A class of isotropic distributions in $\mathbf{R}^{n}$ and their

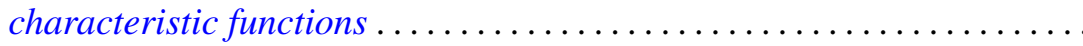

Ezra Brown and Charles John Parry, The 2-class group of biquadratic fields.

II ........................................ 11

Thomas E. Cecil and Patrick J. Ryan, Focal sets of submanifolds ....... 27

Joseph A. Cima and James Warren Roberts, Denting points in $B^{p} \ldots \ldots \ldots 41$

Thomas W. Cusick, Integer multiples of periodic continued fractions . . . . . 47

Robert D. Davis, The factors of the ramification sequence of a class of

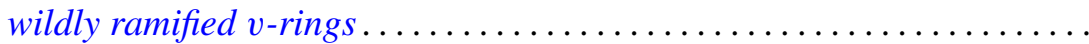

Robert Martin Ephraim, Multiplicative linear functionals of Stein

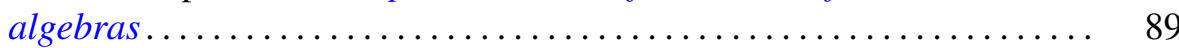

Philip Joel Feinsilver, Operator calculus . .................... 95

David Andrew Gay and William Yslas Vélez, On the degree of the splitting field of an irreducible binomial ..........................

Robert William Gilmer, Jr. and William James Heinzer, On the divisors of

monic polynomials over a commutative ring ..................

Robert E. Hartwig, Schur's theorem and the Drazin inverse .............

Hugh M. Hilden, Embeddings and branched covering spaces for three and four dimensional manifolds ............................

Carlos Moreno, The Petersson inner product and the residue of an Euler product. ...

Christopher Lloyd Morgan, On relations for representations of finite groups....

Ira J. Papick, Finite type extensions and coherence

$\mathrm{R}$. Michael Range, The Carathéodory metric and holomorphic maps on a class of weakly pseudoconvex domains ................

Donald Michael Redmond, Mean value theorems for a class of Dirichlet series

Daniel Reich, Partitioning integers using a finitely generated semigroup ...

Georg Johann Rieger, Remark on a paper of Stux concerning squarefree

numbers in non-linear sequences

Gerhard Rosenberger, Alternierende Produkte in freien Gruppen ..

Ryōtarō Satō, Contraction semigroups in Lebesgue space

Tord Sjödin, Capacities of compact sets in linear subspaces of $\mathbf{R}^{n}$

Robert Jeffrey Zimmer, Uniform subgroups and ergodic actions of exponential Lie groups......................... 